Ann. Zootech., I968, 17 (2), I83-I97.

\title{
UTILISATION COMPARÉE DES CÉRÉALES COMME SEULS ALIMENTS DU PORC PENDANT LA PÉRIODE DE FINITION
}

\author{
Y. HENRY \\ avec la collaboration technique de A. GAye, J.-P. Turc et H. Bousquet \\ Station de Recherches sur l'Élevage des Porcs, \\ Centre national de Recherches zootechniques, 78 - Jouy-en-Josas \\ Institut national de la Recherche agronomique
}

\section{SOMMAIRE}

L'utilisation comparée des céréales (orge, blé, maïs) comme seuls aliments du Porc pendant la période de finition, par référence à un régime témoin équilibré à base d'orge, a fait l'objet de deux expériences, la première sur des animaux en cages de digestibilité, pesant en moyenne $5 \mathrm{I}$ kg et au nombre de 5 par régime, la deuxième sur 4 lots de 6 animaux, entre 60 et $100 \mathrm{~kg}$ de poids vif. L'essai de digestibilité a fait apparaître une liaison très étroite entre la valeur énergétique des régimes et le taux de cellulose dans la ration totale. Les valeurs d'énergie digestible, en kcal par kg de matière sèche de céréale, sont respectivement $3489 \pm 36,385^{8} \pm$ i 8 et $3965 \pm 24$ pour l'orge, le blé et le maïs. Ces résultats sont discutés en relation avec les données de la littérature. Par ailleurs, compte tenu des résultats de croissance et de composition corporelle. les meilleures performances sont obtenues dans le lot témoin, suivi dans l'ordre par l'orge, le blé et le maïs. Les différences en faveur du lot témoin et de l'orge s'expliquent en réalité par un meilleur éq̨uilibre entre la lysine et l'énergie digestible contenue dans le régime. La discussion des résultats met particulièrement l'accent sur l'importance du rapport acide aminé limitant (lysine)/énergie dans la ration, par opposition au rapport azote/énergie qui n'a de signification précise que pour une source azotée donnée.

\section{INTRODUCTION}

Les céréales constituent la base de l'alimentation du porc charcutier. En raison d'un déséquilibre plus ou moins prononcé entre les fractions énergétique et azotée, leur introduction dans le régime n'en nécessite pas moins un apport complémentaire de matières azotées et d'acides aminés indispensables, le plus généralement sous forme de tourteaux ${ }^{*}$ et de farines animales. Cependant, compte tenu des exigences relativement faibles du Porc en acides aminés indispensables pendant la phase de 
finition, il est possible d'envisager durant cette période l'utilisation quasi exclusive des céréales dans la ration, à condition de disposer de variétés suffisamment riches en matières azotées et de procéder à une supplémentation par les acides aminés limitants, parallèlement à un apport complémentaire de minéraux et de vitamines. En même temps qu'a été abordé un programme d'expérimentation sur la supplémentation des céréales seules (orge, blé, maïs) par des aminoacides de synthèse, principalement la lysine (RÁRAT et HENRY in RÉRAT I966, I967), nous avons entrepris de comparer leur efficacité dans le régime du Porc en finition, soit entre 60 et Ioo $\mathrm{kg}$ de poids vif.

\section{MATÉRIEL ETT MÉTHODES}

L'emploi des céréales (orge, blé et maïs) comme seuls aliments du Porc pendant la période de finition, par référence à un régime témoin équilibré, a fait l'objet de deux expériences, l'une en cages de digestibilité et l'autre en lots.

\section{a) Expérience en cages de digestibilité}

L'étude de l'utilisation digestive des éléments énergétiques et azotés de régimes exclusivement constitués de céréales a été réalisée sur 5 groupes de 4 porcs mâles castrés, de race Large White et d'un poids moyen initial de $5 \mathrm{Igg}$. A l'intérieur de chaque groupe, les animaux sont affectés au hasard aux quatre traitements suivants :
A : régime témoin à base d'orge,
B : orge seule,
C: blé scul,
$\mathrm{D}$ : mais seul.

La composition des céréales et des régimes est rapportée dans les tableaux I et 2. Les céréales, préalablement broyées (tamis de $3 \mathrm{~mm}$ ), sont introduites dans la ration au taux de $96 \mathrm{p}$. 1oo et complétées par 4 p. 100 d'un mélange minéral et vitaminique. Les animaux sont nourris suivant la technique paired-feeding de Mitchell (I930). A cet effet, la quantité de nourriture distribuée a été volontairement réduite à $\mathrm{I}, 4 \mathrm{~kg}$ par jour, en 3 repas, en raison des risques importants de refus dans le lot " maïs ». Les régimes sont offerts à l'état humide (trois parties d'eau pour une partie d'aliment $\mathrm{sec})$.

Les méthodes d'étude de la digestibilité ont été décrites dans une publication antérieure (HENRY et RÉRAT, I966). Dans les trois premières répétitions, il a été procédé à une période de collecte de 6 jours; dans les deux dernières, deux périodes de collecte consécutives de 6 jours se sont avérées nécessaires, afin d'améliorer la précision des observations. Les contenus énergétiques des régimes, fèces et urine sont mesurés à l'aide d'un calorimètre adiabatique Gallenkamp. Les échantillons d'urine ont été préalablement séchés sous vide à $25^{\circ} \mathrm{C}$ dans des sacs en polyéthylène, selon la méthode de NiJkamp (I965).

\section{b) Expérience en lots}

Vingt-quatre porcs de race Large While, dont 12 mâles castrés et 12 femelles, sont soumis à une croissance normale jusqu'à $60 \mathrm{~kg}$ de poids vif. Ils sont alors répartis par groupes de 4 d'après le sexe, le poids et l'âge. A l'intérieur de chaque groupe les animaux sont affectés au hasard aux quatre traitements précédents ( $\mathrm{A}, \mathrm{B}, \mathrm{C}, \mathrm{D})$, et élevés en loges individuelles jusqu'au poids de $100 \mathrm{~kg}$. Ils sont nourris selon leur appétit, à raison de trois repas humides par jour (trois parties d'eau pour une partie d'aliment sec); le niveau de la ration journalière est réglé de telle façon que les animaux soient à la limite du refus. Les quantités de nourriture consommées sont enregistrées quotidiennement. Au cours de l'expérience, il est procédé à la pesée des animaux à intervalles réguliers. (tous les I4 jours). Au poids de Ioo kg, ils sont abattus et les carcasses sont découpées selon la technique habituelle (RÉRAT et HENRY, I964). 
TABLEAU I

Composition chimique des céréales

\begin{tabular}{|c|c|c|c|}
\hline & Orge & Blé & Mais \\
\hline Matière sèche $p .100 \ldots \ldots \ldots \ldots \ldots$ & 85,7 & 84,6 & 88,0 \\
\hline $\begin{array}{l}\text { Composition } p .100 \text { matière sèche }: \\
\text { matière organique } \ldots \ldots \ldots \ldots \ldots \ldots \\
\text { matières azotées }(\mathrm{N} \times 6,25) \ldots \ldots \ldots \ldots \\
\text { cellulose brute }(\text { WEENDE) } \ldots \ldots \ldots \ldots \\
\text { énergie brute }(\mathrm{kcal} / \mathrm{kg} \text { matière sèche })\end{array}$ & $\begin{array}{r}97,5 \\
11,4 \\
4,1 \\
4309\end{array}$ & $\begin{array}{r}98,3 \\
11,5 \\
2,2 \\
4431\end{array}$ & $\begin{array}{r}98,0 \\
9,8 \\
1,6 \\
4513\end{array}$ \\
\hline Poids spécifique & 69,4 & 75,2 & 70,2 \\
\hline
\end{tabular}

TABLEAU 2

Composition des régimes

\begin{tabular}{|c|c|c|c|c|}
\hline Régime & $\begin{array}{c}\text { Témoin } \\
\text { "Finition " }(\mathbf{1})\end{array}$ & "Orge " $\left({ }^{2}\right)$ & " Blé \# $\left({ }^{2}\right)$ & " Maïs " $\left.{ }^{2}\right)$ \\
\hline Lot & A & B & $\mathrm{C}$ & $\mathrm{D}$ \\
\hline Matière sèche $\mathrm{p} .100 \ldots \ldots \ldots \ldots$ & 86,1 & 85,5 & 86,5 & 87,4 \\
\hline $\begin{array}{l}\text { Composition de la matière sèche } p .100: \\
\text { matière organique } \ldots \ldots \ldots \ldots \ldots \ldots\end{array}$ & 95,1 & $9 i, 8$ & 95,0 & $9_{1, t_{t}^{\prime}}^{\prime}$ \\
\hline matières azotées $\ldots \ldots \ldots \ldots \ldots \ldots \ldots$ & 15,5 & 10,1 & 12,5 & 9,8 \\
\hline matières grasses ........... & 1,6 & $1, \overline{7}$ & 1,7 & 4,1 \\
\hline cellulose brute (WEENDE) $\ldots \ldots \ldots \ldots$ & 6,8 & 5,7 & 4,4 & 2,7 \\
\hline énergie brute $(\mathrm{kca} / \mathrm{kg}$ matière sèche) .... & 4256 & 1113 & 4228 & ' 31 t' \\
\hline Teneur en lysine (estimée) $\left({ }^{2}\right.$ ) & & & & \\
\hline 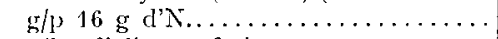 & & 3,73 & 2,70 & 3,79 \\
\hline $\mathrm{g} / \mathrm{kg}$ d'aliment frais... & 6,9 & 3,26 & 2,94 & 2,59 \\
\hline $\mathrm{g} / 1000 \mathrm{kcal}$ digestibles $\ldots$ & 2,05 & 1,13 & 0,92 & 0,80 \\
\hline
\end{tabular}

(1) Composition p. 100 : orge, 83; tourteau de soja, 3; tourteau d'arachide, 3; farine de luzerne, 5; farine de viande, 2; levure de distillerie, $2 ;$ mélange minéral et vitaminique (IIENRY et RÉRAT, 1966), 2;

() Régimes renfermant 96 p.100 de chacune des céréales et ' $\{$ p. 100 du même méla nge minêral et vitaminique que dans le régime témoin.

(3) D'après les valeurs domées par Pion et Falconneav (1966), en fonction du taux de matières azotées de la céréale. 


\section{RÉSULTATS}

\section{a) Digestibilité et rétention azotée}

Les résultats généraux de digestibilité sont résumés dans le tableau 3. Le coefficient d'utilisation digestive (CUI)) apparent de l'énergie est nettement plus élevé avec le blé et le maïs ( $87 \mathrm{p}$. Ioo) qu'avec l'orge et le régime finition (respectivement

\section{TABLEAU 3 \\ Résultats généraux de digestibilité}

Poids vif moyen initial : $5 \mathrm{I}, 2 \mathrm{~kg}$. Poids vif moyen final : $54, \mathrm{r} \mathrm{kg}$

Quantité consommée/j : I,4 kg d'aliment frais

Durée : 2I jours, dont une période préexpérimentale de 7 jours et deux périodes de collecte consécutives de 6 jours.

\begin{tabular}{|c|c|c|c|c|c|}
\hline Régime & "Finition" & "()rge" & "Blée & " Maïs " & $\begin{array}{l}\text { Ecart-type } \\
\text { de la moyenne } \\
s \bar{x}\left({ }^{\mathbf{3}}\right)\end{array}$ \\
\hline Lot & $A$ & 13 & $\mathrm{C}$ & $\mathrm{D}$ & \\
\hline 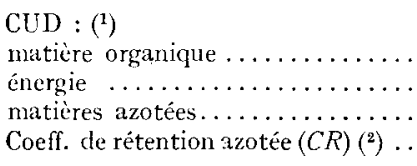 & $\begin{array}{l}81,1 \\
79,0 \\
79,0 a \\
48,8\end{array}$ & $\begin{array}{l}83,8 \\
81,0 \\
74,2 \\
39,0\end{array}$ & $\begin{array}{l}89,1 a \\
87,0 a \\
81,6 a \\
25,5 a\end{array}$ & $\begin{array}{l}89,9 a \\
87,8 a \\
81,6 a \\
21,8 a\end{array}$ & $\begin{array}{ll}0,60 & (1,6) \\
0,54 & (1,4) \\
1,2 \quad(3,3) \\
1,8(12,1)\end{array}$ \\
\hline $\begin{array}{l}\text { Énergie digestible }(\mathrm{kcal} / \mathrm{kg} / \mathrm{MS}) \ldots \\
\text { Énergie métabolisable }(\mathrm{kcal} / \mathrm{kg} \text {.IIS) } \\
\text { Énergie métabolisable } \mathrm{p} .100 \\
\text { Énergie digestible } \ldots \ldots \ldots \ldots \ldots \ldots\end{array}$ & $\begin{array}{l}3363 a \\
: 248 a \\
96,75 a\end{array}$ & $\begin{array}{l}3331 a \\
3 \cdot 2-27 a \\
97,17 b\end{array}$ & $\begin{array}{l}3681 \\
3557 \\
96,76 a\end{array}$ & $\begin{array}{r}3762 \\
3638 \\
97,04 b\end{array}$ & $\begin{array}{l}214(1,5) \\
24(1,6) \\
0,12(0,27)\end{array}$ \\
\hline Énergie urine (kcal/g d'azote)..... & $10,95 a$ & 12,10 & 9,28 & $11,14 a$ & $0,29 \quad(6,0)$ \\
\hline 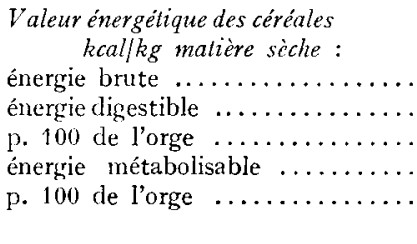 & & $\begin{array}{c}4358 \\
3489 \pm 36(3) \\
3380 \pm \frac{13}{100}\end{array}$ & $\begin{array}{c}4328 \\
3858 \pm 18 \\
111 \\
3728 \pm 20 \\
110\end{array}$ & $\begin{array}{c}4642 \\
3965 \pm 24 \\
114 \\
3835 \pm 24 \\
113\end{array}$ & \\
\hline
\end{tabular}

() CUD : (élément ingéré-élément excrété (fèces)) $\times 100 /$ élément ingéré.

$\left({ }^{2}\right) \mathrm{N}$ retenu $\times 100 / \mathrm{N}$ absorbé.

${ }^{(3)}$ Écart-type de la valeur moyenne pour 5 animaux : $s \bar{x}$; le coefficient de variation est indiqué entre parenthèses. Les moyennes affectées d'un même indice ne sont pas significativement différentes entre elles au seuil 0,05 : test de comparaison multiple des moyennes utilisant les tables de PEArson et HartLEy (1966).

$8 \mathrm{r}$ et $79 \mathrm{p}$. Ioo). Les valeurs des CUD des matières azotées sont respectivement : $86-8 \mathrm{I}, 6-79$ et 74,2 pour le blé, le maïs, le régime finition et l'orge. I'utilisation diges- 
tive des éléments énergétiques et azotés des céréales est ainsi en relation inverse avec le pourcentage de constituants cellulosiques, comme le montre la figure I. Malgré un nombre réduit de données, correspondant seulement à 4 taux de cellulose inégalement espacés, il a été procédé à un calcul de régression du CUD de l'énergie sur le tatıx de cellulose brute rapporté à la matière sèche $(x)$. Ce calcul fait apparaitre une décroissance linéaire du CUD de l'énergie en fonction du taux de cellulose, suivant la relation :

$$
\begin{gathered}
\mathrm{CUD} \cdot \mathrm{E}=92,45-2,39 \times \text { (taux de cellulose } \mathrm{p} \text {. roo matière sèche), } \\
\text { le coefficient de corrélation étant } 0,90 .
\end{gathered}
$$

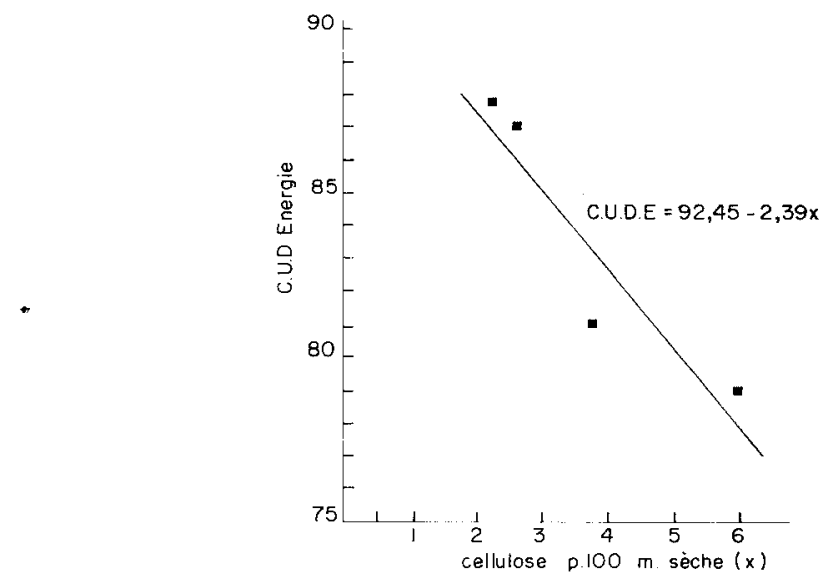

Fig. I. - Relation entre le CUD de l'énergie et le taux de cellulose du régime

A partir des valeurs d'énergie digestible de la matière organique des régimes, nous avons déduit les valeurs énergétiques des céréales pures, soit respectivement : 3489 , 3858 et $3965 \mathrm{kcal}$ par $\mathrm{kg}$ de matière sèche pour l'orge, le blé et le maïs. L'énergie métabolisable apparente représente une proportion sensiblement constante de l'énergie digestible (96 à 97 p. roo). Notons cependant que l'énergie excrétée dans l'urine a tendance à être plus faible dans le lot " orge " que dans les lots "finition " et "blé » (différence significative au seuil o,Io). Par ailleurs, le contenu énergétique de l'urine, exprimé en $k c a l$ par $g$ d'azote, varie d'une façon significative d'un lot à l'autre ; ceci est particulièrement net pour le blé, dont les valeurs observées sont systématiquement plus faibles que pour les autres céréales.

En ce qui concerne le coefficient de rétention azotée, la valeur la plus élevée est obtenue avec le régime témoin ( $48,8 \mathrm{p}$. Ioo). Ensuite, vient l'orge (39,0 p. Ioo), suivie de très loin par le blé $(25,5$ p. IOO) et le maïs $(2 \mathrm{I}, 8 \mathrm{p}$. I00). Il faut en conclure que les protéines de blé et de maïs sont nettement plus déséquilibrées que celles de 1'orge. En comparant les teneurs en acides aminés des céréales aux besoins du Porc pendant la phase de finition, il apparait, en effet, que la lysine constitue le facteur limitant primaire de ces dernières, les quantités pour I6 $\mathrm{g}$ d'azote étant respectivement 3,73-2,70 et 3,09 pour l'orge, le blé et le maïs (tabl. 2). Il importe toutefois d'interpréter ces valeurs de rétention azotée avec une certaine prudence, compte tenu des 


\section{TABIEAU 4}

Résultats généraux de croissance, consommation

Poids moyen initial : $60,8 \mathrm{~kg}$ Poids moyen final : 98,8 kg Nombre d'animaux par lot : 6

\begin{tabular}{|c|c|c|c|c|c|}
\hline Régime (1) & $\begin{array}{l}\text { Témoin } \\
\text { "Finition" }\end{array}$ & "Orge " & « Blé » & "Maĩs " & Écart-type \\
\hline Lot & A & $\mathrm{B}$ & C & $\mathrm{D}$ & \\
\hline $\begin{array}{l}\text { Durée (jours) } \ldots \ldots \ldots \ldots \ldots \ldots \ldots \\
\text { Gain moyen }(j \mid g) \ldots \ldots \ldots \ldots \ldots \ldots\end{array}$ & $\begin{array}{ll}47,8 & \\
828 & a\end{array}$ & $\begin{array}{cc}53,7 & \\
733 & a\end{array}$ & $\begin{array}{cc}60,7 & \\
618 & b\end{array}$ & $\begin{array}{c}64,2 \\
596\end{array}$ & $38(13,4)$ \\
\hline Consommation/j: & & & & & \\
\hline aliment (poids frais, $\mathrm{kg}$ ) ...... & $3,34 a$ & $3,30 a$ & $2,97 b$ & $3,16 a b$ & $0,09(7,0)$ \\
\hline $\begin{array}{l}\text { énergie digestible, kcal ........ } \\
\text { matières azotées digestibles }(\mathrm{g}) \\
\text { lysine }(\mathrm{g}) \ldots \ldots \ldots \ldots \ldots \ldots \ldots\end{array}$ & $\begin{array}{c}9800 \quad a \\
355 \\
20,0\end{array}$ & $\begin{array}{c}9520 \quad a \\
214 \\
10,8\end{array}$ & $\begin{array}{c}9510 \quad a \\
278 \\
8,7\end{array}$ & $\begin{array}{c}10190 \quad a \\
216 \\
8.2\end{array}$ & $224(5, f)$ \\
\hline $\begin{array}{l}\text { Indice de consommation : } \\
\quad \mathrm{kg} \text { d'aliment } / \mathrm{kg} \text { de gain } \ldots \ldots . \\
\mathrm{kcal} \text { dig. } / \mathrm{kg} \text { de gain } . . . \ldots \ldots\end{array}$ & $\begin{array}{l}4,03 a \\
11820 a\end{array}$ & $132 \longleftarrow 0^{4,59} a b$ & $\begin{array}{l}4,89 b c \\
15660^{\circ}\end{array}$ & $\begin{array}{r}5,38 c \\
17470\end{array}$ & $\begin{array}{l}0,21(1,1) \\
662(1.1)\end{array}$ \\
\hline
\end{tabular}

(1) Les régimes $\mathrm{A}, \mathrm{B}, \mathrm{C}, \mathrm{D}$, utilisés dans l'expérience en lots, renfermaient respectivement : $87,2-86,6$ $-87,0$ et 85,7 p. 100 de matière sèche $; 14,5-8,7-10,9$ et 8,4 p. 100 de matières azotées.

() Voir légendes du tableau 2.

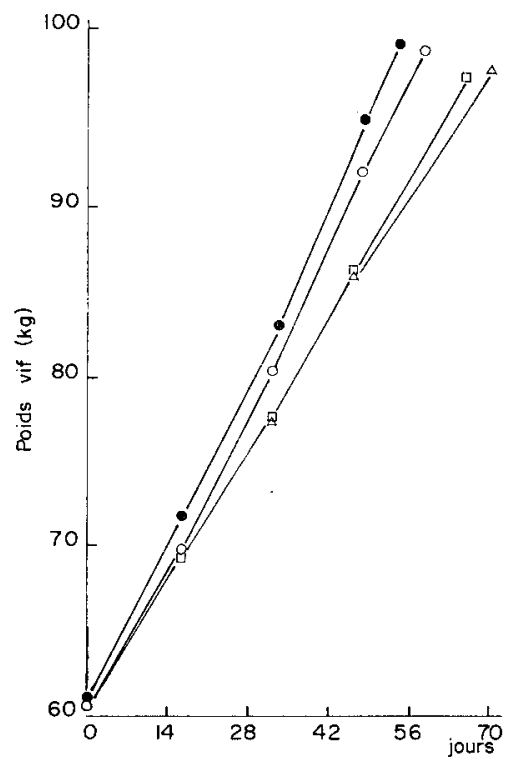

Fig. 2. - Courbes de croissance

•-_ Témoin Finition o—o Orge $\square \longrightarrow \square$ Blé $\triangle \longrightarrow \_$Mais 
difficultés rencontrées pour égaliser l'apport journalier de nourriture à un niveau compatible avec une vitesse de croissance satisfaisante ; cette dernière a été respectivement de $240,190,270$ et $240 \mathrm{~g} /$ jour dans les lots $\mathrm{A}, \mathrm{B}, \mathrm{C}$ et $\mathrm{D}$, pendant les périodes de collecte.

L'analyse statistique des résultats de digestibilité sur les deux dernières séries d'animaux, qui comportaient chacune 2 périodes de collecte successives, a permis de séparer dans la variation résiduelle, la part de variation entre périodes intra-animal (ou erreur analytique) et la part de variation entre animaux (ou erreur individuelle). Ces deux sources d'erreur, exprimées en coefficient de variation, sont sensiblement du même ordre de grandeur; par exemple, lorsque l'on prend en considération le CUD de l'énergie, elles représentent respectivement I, I et I,4 p. Ioo de la valeur moyenne. Ceci indique que pour une même précision expérimentale, on a avantage à multiplier le nombre d'animaux par traitement plutôt que le nombre d'observations par animal. Quoi qu'il en soit, il est bon de prévoir deux périodes de collecte par animal si l'on désire contrôler l'erreur analytique.

\section{b) Croissance, consommation et composition corporelle}

Comme l'indiquent le tableau 4 et la figure 2 concernant l'évolution du poids vif, les meilleures performances de croissance sont obtenues dans le lot " témoin finition ", suivi dans l'ordre par les lots "orge ", "blé " et " maïs ". Remarquons à cet effet que les 4 lots se séparent en 2 groupes ('Témoin-Orge) et (Blé-Maïs), significativement différents entre eux, non seulement du point de vue de la croissance, mais également du point de vue de la valeur énergétique, elle-même fonction du taux de cellulose; dans tous les cas, la vitesse de croissance observée est en relation inverse avec la valeur énergétique du régime, tout au moins dans nos conditions expérimentales.

Les écarts de croissance enregistrés selon la nature de la céréale s'expliquent en fait par des différences dans le niveau d'ingestion, qu'il s'agisse des résultats moyens ou de leur évolution dans le temps en fonction du poids vif moyen par quinzaine (fig. 3 a). Ainsi, la quantité de nourriture consommée est sensiblement la même dans les lots "finition " et "orge " et nettement plus élevée que dans les lots "maïs" et "blé ". Exprimée en kcal digestibles, la consommation d'énergie est la plus faible dans les lots " orge " et "blé " et la plus élevée dans le lot " maïs " (fig. $3 b$ ). Du point de vue de 1'indice de consommation, exprimé en kcal digestibles par $\mathrm{kg}$ de gain, les régimes se classent dans l'ordre inverse de celui du gain moyen journalier, l'indice le plus favorable étant obtenu avec le régime témoin " finition ».

L'évolution de la consommation journalière de matières azotées digestibles en fonction du poids vif est précisée dans la figure $3 c$. Les animaux au maïs et au blé ingèrent sensiblement la même quantité de matières azotées quel que soit leur poids mais nettement moins que ceux recevant l'orge et a fortiori le régime témoin. En réalité, si l'on prend en considération l'acide aminé limitant de chacune dès trois céréales, en 1'occurence la lysine, on s'aperçoit que c'est 1'orge qui fournit l'apport journalier de lysine le plus élevé, puis le blé et le maïs (fig. $3 d$ ), cette différence en faveur de l'orge réside dans un rapport lysine (ou acide aminé limitant) /énergie plus élevé. 

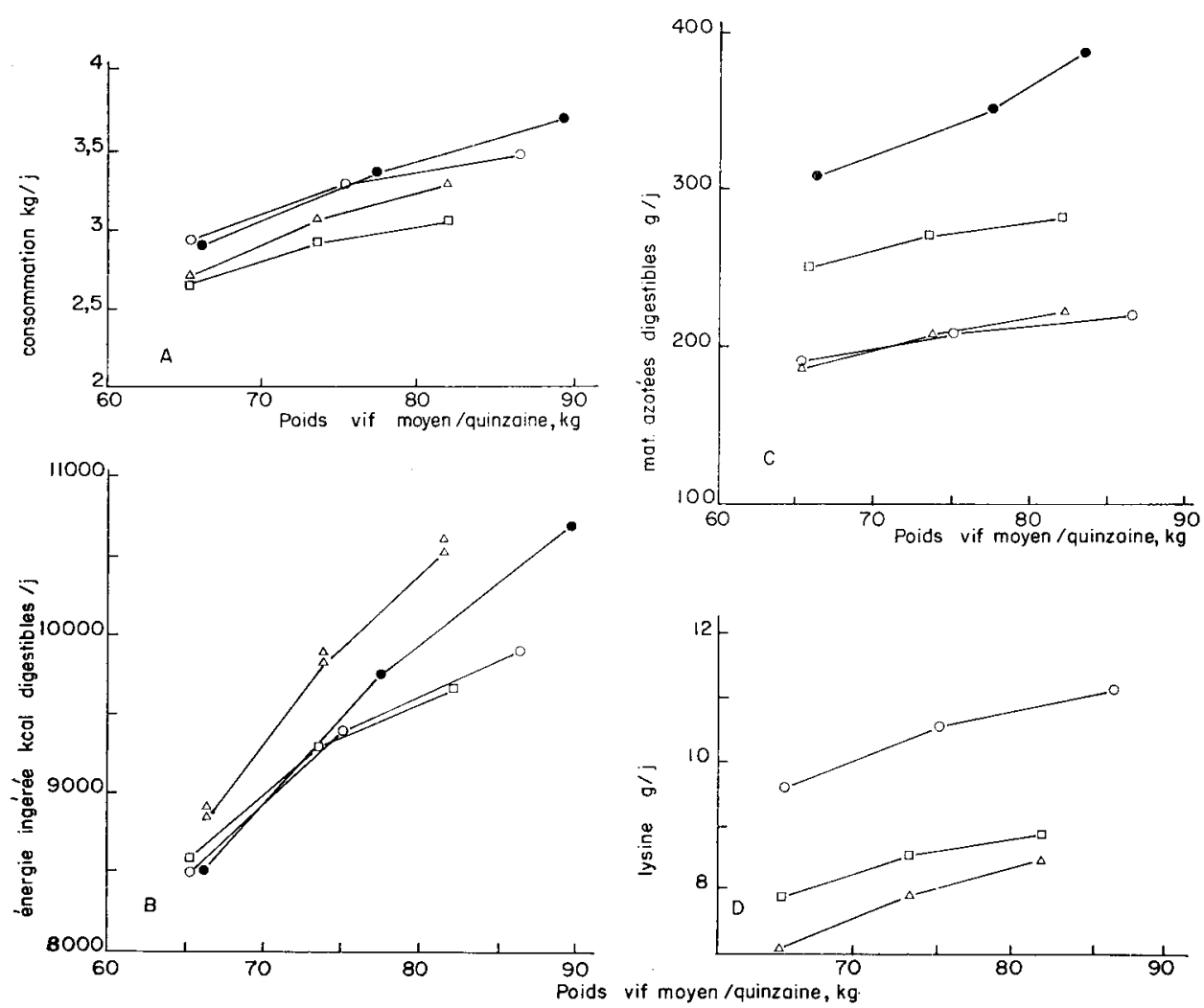

FIG. 3.-- Évolution des consommations journalières en fonction du poids rif moyen par quinzaine
A : Consommation d'aliment, $\quad \mathrm{kg} / \mathrm{j}$
$\mathrm{B}$ : Énergie digestible, $\mathrm{kcal} / \mathrm{j}$
$\mathrm{C}:$ Matières azotées digestibles, $\quad \mathrm{g} / \mathrm{j}$
D: Lysine,
$\mathrm{g} / \mathrm{j}$

- - Témoin Finition o-—o Orge $\quad$ - $-\square$ Blé $\quad \ldots \Delta$ Maïs

Les résultats de composition corporelle (tabl. 5) ne sont d'ailleurs que la conséquence directe des variations de ce rapport lysine/énergie. Ainsi les animaux recevant le régime témoin accusent un rendement à l'abattage et un pourcentage de morceaux gras dans la carcasse plus faibles que ceux nourris de céréales seules, tandis que le pourcentage de morceaux maigres est plus élevé. De la comparaison des céréales entre elles, il résulte que l'orge donne les carcasses les plus maigres, suivie par le blé et le maïs. Par contre, aucune différence n'apparaît dans le poids des viscères. En définitive, il est intéressant de constater que la vitesse de croissance, et d'une manière plus précise, la production de morceaux maigres, est en relation directe avec la quantité de lysine par I ooo kcal digestibles dans la ration (fig. 4). 
TABLEAU 5

Résultats de composition corporelle

Poids moyen final : $98,8 \mathrm{~kg}$

\begin{tabular}{|c|c|c|c|c|c|}
\hline Régime & $\begin{array}{c}\text { Témoin } \\
\text { "Finition " }\end{array}$ & "Orge " & "Blé ") & "Maïs & $\begin{array}{l}\text { Ecart-type } \\
\text { de la moyenne } \\
s \bar{x}(\mathbf{1})\end{array}$ \\
\hline Lot & A & $\mathrm{B}$ & $\mathrm{C}$ & D & \\
\hline 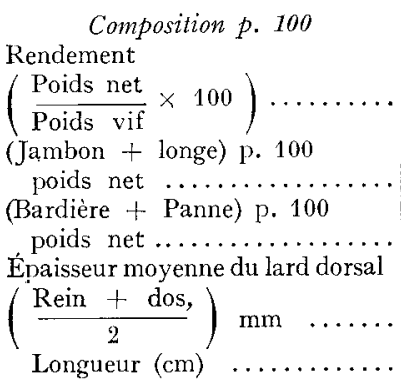 & $\begin{array}{l}50,8 a \\
19,4 a\end{array}$ & $\begin{array}{l} \pm 8,8 a, b \\
22,4 a\end{array}$ & $\begin{array}{l}47,6 \quad b \\
22,7 a \\
35,1 a \\
94,7 a\end{array}$ & $\begin{array}{l}47,0 b \\
23,4 a \\
35,3 a \\
95,7 a\end{array}$ & $\begin{array}{lr}0,80 & (2,7) \\
0,81 & (4,1) \\
3,51 & (8,6) \\
& \\
1,93 & (12,6) \\
1,1 & (2,9)\end{array}$ \\
\hline 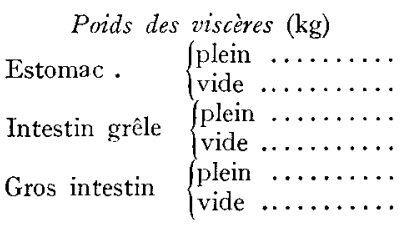 & $\begin{array}{l}2,18 \\
0,68 a \\
3,03 \\
1,68 a \\
4,00 \\
1,99 a\end{array}$ & $\begin{array}{l}1,90 \\
0,66 a \\
2,6^{\prime} \\
1,52 a \\
3,94 \\
1,95 a\end{array}$ & $\begin{array}{l}1,82 \\
0,61 a \\
2,40 \\
1,73 a \\
3,42 \\
1,93 a\end{array}$ & $\begin{array}{l}2,18 \\
0,70 a \\
2,21 \\
1,60 a \\
3,75 \\
2,11 a\end{array}$ & $\begin{array}{l}0,035(11,9) \\
0,102(15,3)\end{array}$ \\
\hline
\end{tabular}

(1) Voir légendes du tableau 2.

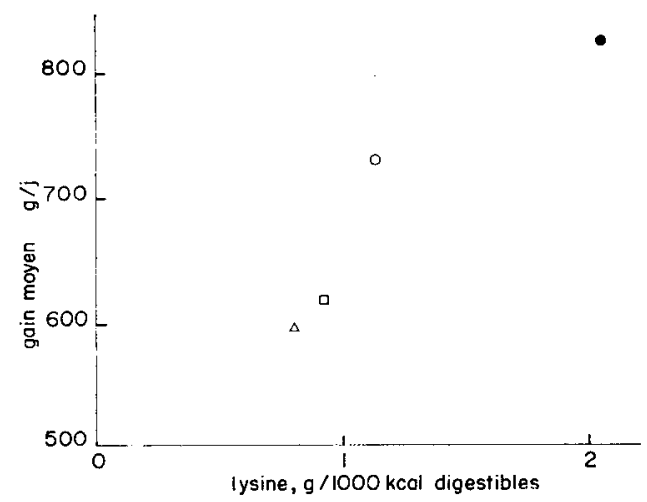

FIG. 4. - Relation entre le gain moyen journalier et la quantité de lysine en $g$, pour 1000 kcal digestibles

- Témoin Finition oOrge $\square$ Blé $\Delta$ Mais 


\section{DISCUSSION}

L'examen des résultats de digestibilité des matières azotées fait ressortir une différence très marquée entre l'orge (74 p. Ioo) et le maïs $(82$ p. Ioo) et surtout le blé (86 p. I00), ce qui est conforme aux données classiques de la littérature (SchNeiderR, I947). Il convient cependant de nuancer cette constatation, en raison des différences de concentration des matières azotées dans les régimes. Parallèlement à 1'utilisation digestive globale des matières azotées, il a été observé par ailleurs une différence importante dans la disponibilité de la lysine : cette dernière serait moins élevée dans l'orge que dans le maïs (NrELSEN et al., I963).

Un deuxième point concerne l'utilisation digestive des substances énergétiques. Depuis les travaux d'Axelsson (I955), Poni) et al. (r962), puis de NEHRing (I966) et plus récemment de DrenNan et Maguirs: (I966), il est maintenant bien établi que les variations du coefficient d'utilisation digestive (CU1)) des éléments énergétiques de la ration, chez le Porc, peuvent être expliquées, pour la plus grande part, par la proportion de matériaux cellulosiques contenus dans le régime. Il est intéressant de remarquer que dans le cas présent, le CUD de l'énergie diminue d'environ 2 p. Ioo lorsque le taux de cellulose brute augmente de I p. Ioo d'une céréale à l'autre, ce qui correspond sensiblement aux résultats trouvés par les auteurs précédents avec des régimes à base de céréales. Quoi qu'il en soit, pour une céréale donnée, les fluctuations de la valeur énergétique sont relativement faibles, comme le prouvent les différentes valeurs trouvées dans la littérature (tabl. 6). I1 faut en conclure que les variations intervenues dans la composition chimique des céréales ou dans les méthodes de détermination de leur valeur énergétique, ont eu relativement peu de répercussions sur l'estimation de cette dernière, tout au moins lorsqu'elle est exprimée en énergie digestible. Il en va sensiblement de même des valeurs d'énergie métabolisable apparente, dont les variations sont toutefois sous la dépendance des taux respectifs de matières azotées et d'acides aminés indispensables dans la ration. Ceci constitue un argument de plus en faveur de la nécessité de déterminer la valeur énergétique des régimes dans les conditions optimum d'apport des acides aminés, ainsi qu'en témoignent les valeurs obtenues sur Rat par VERMOREL et KELLER (I967).

La comparaison du régime témoin de finition et des régimes exclusivement à base de céréales a permis de mettre en évidence dans ces dernières, un déficit azoté à la fois quantitatif et qualitatif, qui se traduit par des performances de croissance réduites et un développement excessif des dépôts gras dans la carcasse. L'influence favorable d'une supplémentation des céréales seules par la lysine (facteur limitant primaire) chez le Porc, a d'ailleurs été démontrée par un certain nombre d'auteurs, qu'il s'agisse de 1'orge (Dinusson, Ig62; RoBINSON et LEWIS, I963; SoldDEvilla et MEADE, I964; RÉRAT, I966, I967); du blé (ERICSON et al., I962; JENSEN et al., I967; RÉrat et HENRY, données non publiées); ou du maïs (POND et JoNEs, I964; POND et $a l$., I964). D'un autre côté, la comparaison des céréales seules, pendant la phase de finition, a fait ressortir un avantage très net de l'orge par rapport au blé et au maïs, en raison précisément d'un meilleur équilibre entre 1'acide aminé limitant (lysine) et le contenu énergétique de la céréale; à noter que TERroINE et VALLA 
avaient déjà montré, en r933, que l'orge constitue la meilleure céréale pour le porc, du point de vue de la qualité de ses protéines. Non seulement 1'orge présente une teneur plus élevée en lysine $(3,9 \mathrm{~g}$ par $\mathrm{kg}$ de matière sèche contre 3.5 et 3,2 pour le blé et le maïs), mais encore, elle est moins riche en énergie (3489 kcal digestibles par kilo de matière sèche contre 3858 et 3965 pour le blé et le maïs), ce qui se traduit par une consommation accrue de nourriture, donc de lysine, alors que le niveau d'ingestion d'énergie utilisable est plus faible. L'amélioration des performances de croissance et de carcasses avec le blé et le maïs, dans les conditions d'alimentation selon l'appétit, exigerait donc à la fois :

- une diminution de la valeur énergétique du régime, grâce à l'introduction d'un diluant cellulosique ;

- une quantité suffisante de lysine relativement à l'énergie grâce à un apport complémentaire d'une source azotée riche en lysine.

$L_{1}$ a dilution du contenu énergétique de la céréale peut sans doute être obtenue à l'aide d'un matériau cellulosique quelconque (rafles, fourrages grossiers), mais compte tenu de la nécessité d'un apport complémentaire de 1ysine, il apparaît plus judicieux d'associer à une céréale énergétique, comme le blé ou le maïs, un complément à la fois riche en acides aminés indispensables, principalement en lysine, et relativement peu énergétique, en l'occurence une farine de luzerne déshydratée par exemple. Dans le même ordre d'idée, il semble peu avantageux de maintenir dans une céréale, comme l'orge, un taux élevé de matériaux cellulosiques, ce qui limite d'autant la possibilité de recourir à certains aliments de complément riches en lysine, mais d'une teneur relativement élevée en cellulose. En définitive, il ressort des observations précédentes que le rapport azote/énergie, qu'on peut exprimer par exemple par la quantité de matières azotées digestibles pour I ooo kcal d'énergie digestible, n'a de signification réelle que s'il se rapporte à une source azotée donnée ; il ne permet aucune discrimination entre des régimes constitués de protéines de qualité différente. Dans tous les cas, au contraire, les résultats de croissance et de composition corporelle sont en relation directe avec le rapport acide aminé limitant (lysine) / énergie, qu'on peut exprimer par la quantité d'acide aminé (lysine), en g pour I ooo kcal d'énergie digestible.

Si l'on considère 1'évolution des résultats de consommation en fonction de la nature de la céréale, on s'aperçoit que d'une manière générale, le niveau d'ingestion varie en raison inverse de la valeur énergétique du régime, conformément à ce que 1'on observe habituellement chez le Porc (HENRy, I966) et chez le Rat (HENRy et RÉRA'T (r966). Cependant, cette relation n'est pas systématique. Ainsi, le maïs est consommé en plus grande quantité que le blé, malgré une valeur énergétique légèrement supérieure. En réalité, la quantité de nourriture librement ingérée par l'animal est, tout au moins pour une large part, la résultante des influences réciproques de la concentration en principes énergétiques et du taux de matières azotées ou d'acides aminés indispensables. A l'appui d'observations effectuées sur le Rat (HENRY et RÉRAT, r965) et sur le Porc (RÉRAT et HENRY, I964), il semblerait que l'animal en croissance cherche à satisfaire, non seulement son besoin énergétique, mais également, dans la mesure du possible, son besoin azoté ; de ce fait, il réagit à un déséquilibre par défaut de protéines vis-à-vis de l'énergie (régime hypoazoté) en élevant son niveau d'ingestion, et ceci d'autant plus que le déséquilibre est plus prononcé. On peut expliquer de cette façon les différences de consommation entre maïs et blé en 


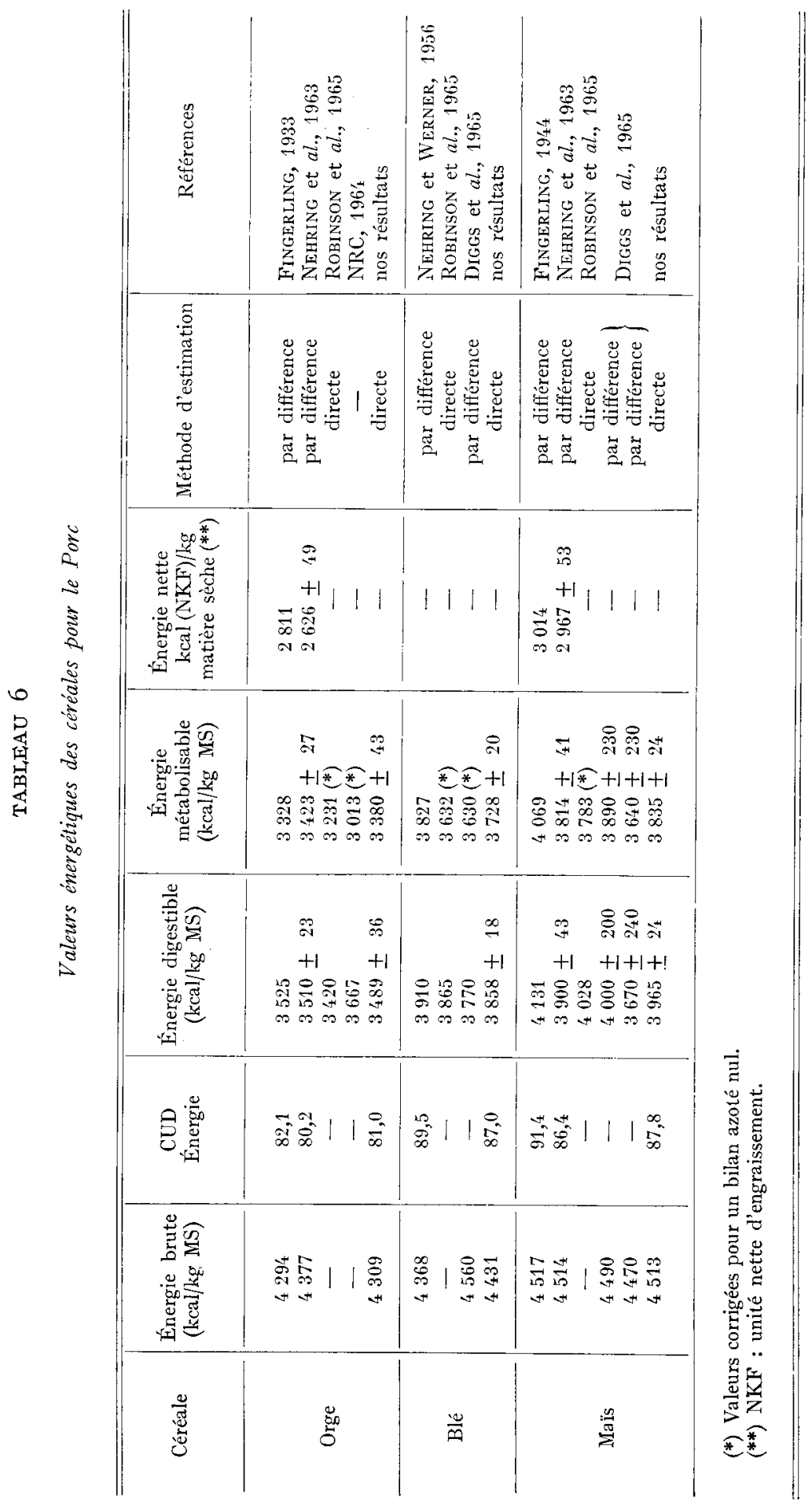


relation simplement avec le taux de matières azotées, nettement plus faible dans le maïs que dans le blé. On peut se demander également si la consommation relativement réduite de blé par rapport aux deux autres céréales ne pourrait pas être attribuée au mode de présentation des régimes. Si l'on se réfère aux récentes observations de LAWRENCE (I967), il apparaîtrait que le Porc accepte plus volontiers le blé lorsqu'il est présenté sous forme aplatie qu'à l'état de farine, comme c'est le cas dans la présente étude.

En conclusion, l'utilisation des céréales seules par le Porc pour la croissance est en relation directe avec le rapport lysine /énergie de la céréale, les meilleures performances étant obtenues avec l'orge, suivie par le blé, puis le maïs. L'amélioration des conditions d'emploi des régimes à base de céréales, chez le Porc, implique ainsi un réajustement de l'apport du ou des acides aminés limitants relativement à l'énergie, grâce à une supplémentation par des sources protidiques riches, accompagnée ou non d'une dilution de l'énergie de la céréale par un aliment cellulosique.

\section{Rę̧u pour publication en mars 1968.}

\section{SUMMARY}

COMPARATIVE UTILIZATION OF CEREALS AS THE ONLY FEED

BY PIGS IN THE FINISHING PERIOD

Comparative utilization of cereals (barley, wheat, maize), in relation to a balanced control died based on barley, as the only feed for pigs in the finishing period, was the subject of 2 experiments each with 4 groups of Large White pigs, given, respectively :

- a control diet based on barley, oilmeals and animal protein meals, with 14.5 per cent crude protein (Group A).

- diets with 96 per cent of one of the three cereals, barley (Group B), wheat (Group C) or maize (Group D) and 4 per cent minerals and vitamins to complete the diet.

Utilization of energy and nitrogen was studied in a digestibility trial lasting 2 I days with castrated males of average initial weight $5 \mathrm{I} \mathrm{kg}$. The pigs were pair-fed and were given $\mathrm{x} .4 \mathrm{~kg}$ feed per day. day. The second experiment was with groups of 6 pigs, half castrated males and half females, kept in individual pens between 60 and $100 \mathrm{~kg}$ liveweight and given moist feed to appetite in 3 meals a day.

The results for digestibility show a very close relation between the energy value and the fibre content of the whole ration (fig. I) : digestibility of energy fell by 2.3 percentage units when the crude fibre (Weende) in the dry matter increased by i per cent. Values for digestible energy, in $\mathrm{kcal}$ per $\mathrm{kg}$ dry matter of the cereal, were $3489 \pm 36,3858 \pm 18$ and $3965 \pm 24$ for barley, wheat and maize, respectively, corresponding to apparent digestibility of energy of $81.0 ; 87.0$ and 87.8 per cent (table 3). Apparent digestibility of crude protein was higher for wheat (86.0) and maize (8I.6) than for barley (74.2) and the control diet (79.0). The results are discussed in relation to data from the literature (table 6).

Considering the results for growth and body composition (tables 4 and 5 ), the best performances were in the control group (A), followed by barley (B), wheat (C) and maize (D) in that order. Respective daily gains were $828,733,6 \mathrm{r} 8$ and $596 \mathrm{~g}$; daily intakes of feed were $3.34,3.30,2.97$ and $3.16 \mathrm{~kg}$, and intakes per $\mathrm{kg}$ gained were $4.03,4.59,4.89$ and $5.38 \mathrm{~kg}$. As proportion of carcass weight ham and loin were 50.8, 48.8, 47.6 and 47.0 per cent and back fat and kidney fat were 19.4, 22.4, 22.7 and 23.4 per cent. The difference in favour of the control diet and barley could be explained in fact by the better balance between lysine and digestible energy in the diet; the diets had $2.05,1.13,0.92$ and $0.80 \mathrm{~g}$ lysine per $\mathrm{I} 000 \mathrm{kcal}$ digestible energy for groups $\mathrm{A}, \mathrm{B}, \mathrm{C}$ and $\mathrm{D}$, respectively. 


\section{RÉFÉRENCES BIBLIOGRAPHIQUES}

Axeisson J., i955. Der Einfluss des Rohfaser gehaltes des Futters auf das Wachstum von Jungschweinen. Arch. Tierernährung, 5, I-16.

Diggs B. G., Becker D. E., Jensen A. H., Norton H. W., 1965. Energy value of various feeds for the young pig. J. Anim. Sci. 24, 555-558.

Dinusson W. E., Erikson D. O., Hacgse C. N., Bolin D. W., 1962. Barley rations for swine : protein and lysine as supplements. North Dakota Res. Rep., no 5 .

Drennan P., Maguire M. F, i966. Relationship pf metabolisable energy in pig feeds to composition. An Foras Taluniais Animal Productoon Res. report, I $13-114$.

Ericson L. E., Larsson S., Óstholm C. O., 1962. Preliminary studies on the possibility of improving the protein value of cereals for swine by lysine supplementation. Acta Agric. Scand., 12, I57-166.

Fingerling G., 1933. Der Stärkewert des Gerstenschrotes. Landwirlsch Versuchs-Stal, 116, r-63.

Fingerling G., I944. Z. Tieremähr. u. Futtermittelkd, 8, i. cité par Becker M., et Neiring K. IIandbuch der Futtermittel, tome 2, p. I 70, Parey ed., Hamburg, 1965.

HenkY Y., I966. Variations des taux énergétique et azoté du régime chez le Porc en croissance. IX'Congrès International de Zootechnie, Édimbourg.

HENRY Y., RÉrAT A., I965. Ingestion spontanée d'éléments énergétiques en régimes mixtes et séparés chez le Rat en croissance. Ann. Biol. anim. Bioch. Biophys., 5, 283-292.

HENRY Y., RÉRAT A, I966. Utilisation des pommes de terre déshydratées et frâiches dans l'alimentation du Porc en croissance en comparaison avec l'orge. Ann. Zootech., 15, 23 I-25I.

HeNRY Y., RÉrat A., I966. Évolution de l'ingestion spontanée de principes énergétiques en fonction de la vitesse de croissance et de la protéinogénèse chez le Rat blanc. Aminoacides, Peptides, Protëines, Cahier $n^{\circ}$ 6, 240-262, A. E. C., Paris.

Jensen A. H., Becker D. E., IIArmon B. G., Ig67. Wheat in diets for funishing swine. J. Anim. Sci., 26, I 473 (abstr.)

LAwrence T. L. J., 1967. High level cereals diets for the growing finishing pig. I. The effect of cereal preparation and water level on the performance of pigs fed diets containing high levels of wheat. J. Algric. Sci., 68, 269-274.

Mitchell H. H., I930. The paired-feeding method : its value and limitations in livestock experimentation. Proc. Amer. Soc. Anim. Prod., 63-73.

National Research council. (NRC), I964. Joint United States-Canadian Tables of feed composition:Nutritional Data for U.S.A. and Canadian feeds $167 \mathrm{pp}$., National Academy of Sciences-X.R.C, Washington D. C.

Nenring K., 1966. Rohfaser oder rohcellulose. Ein Beitrag zur Entwicklung der Weender luttermittelanalyse. Arch. Tierernähr, 16, 77-102.

Neirking K., Hoffminn L., Schiemand R., ig63. Die energetische Verwertung der Futterstoffe. 3. Mitteilung. Die energetische verwertung der Kraftfutterstoffe durch Schweine. Arch. Tierernähr, 13, I $47-16 \mathrm{I}$.

NeIring K., Werner A., I 956. Wiss. Abhl. Dt. Akad. Landatrtschadtswiss. V/I. Cités par Becker M. et Nehring K., Handbïch der Futlermittel, tome 2, p. r7o, Parey ed., Hamburg, 1965.

Nielsen H. E., llays V. W., Speer V. C, Catron D. V., I963. Lysine supplementation of con and barley base diets for growing finishing swine. J. Anim. Sci., 22, $454^{\circ} 457$.

NiJkAMP, I964. Some remarks about the determination of the heat of combustion and the carbon content of urine. In Blaxter K. L., Lnergy metabolism, 440 pp., Acad. Press, New York.

Pearson F. S., Hartley H. O., 1966. Biometrika tables for slalisticians vol, I, 19I-I93, Cambridge Univ. Press.

Pion R., Fauconneau G., ig66. Les acides aminés des protéines alimentaires. Méthodes de dosage et résultats obtenus. Aminoacides, Peptides, Crotéines, cahier $n^{\circ} 6$, I 58 - . 75, A.E.C., Paris.

Pond W. G., Jones J. R., 1964. Aminoacid supplementation of com diets for finishing pigs. Feedstuffs, 36 (4I), 42-44.

Pond W. G., Kroening G. H., Galio J. T., i964. Aminoacids for swine : dietary requirements for growth and carcass leanness. Proc. Cornell Nutr. Conf. for feed Manufacturers, 50-6r.

Pond W. G., Lowrey R. S., Maner J. H., i962. Effect of crude fiber level on ration digestibility and performance in growing-finishing swine. J. Anim. Sci., 21, 692-696.

RÉRAT A., I966. Utilisation des acides aminés obtenus par voie industrielle dans l'alimentation du Porc en croissance. IX $\mathrm{e}$ Congrès international de Zootechnie, Édimbourg.

RÉRAT A., I967. Utilisation des aminoacides de synthèse dans l'aluimentation du Porc en croissance. Perspectives offertes par leur emploi dans la formulation des régimes pour porcs. L'Alimenlation et la Vie(sous presse).

RÉrat A., Henry Y., I964. Utilisation de la betterave " danoise " dans l'alimentation du Porc en croissance. I. Recherche d'un plan de rationnement. Ann. Zootech., 13, 217-236. 
Rérat A., Henry Y., Ig64. Consommation spontanée d'énergie en alimentation mixte et séparée chez le Porc en croissance. Ann. Biol. anim. Biochem. Biophys., 4, 44I-444.

Robinson D. W., Lewis D., I963. Aminoacid supplementation of a barley ration for the pig. J. Sci. Food. Agric., II, 806-813.

Robinson D. W., PrescotT J. H. D., Lewis D., 1965. The protein and energy nutrition of the bacon pig. IV. Digestible energy values of cereals in pig diets. J. Agric. Sci., 64, 59-66.

SCHNEIDER B. H., I947. Feeds of the world. Their digestibility and composition, 299 pp, Agricultural Experiment Station, West Virginia University, Morgantown.

Soldevila M., Meade R. J., I964. Barley rations for swine. 2. The influence of L-lysine and DL-methionine supplementation of barley soybean meal diets upon rate and efficiency of gain and upon nitrogen retention of growing swine. J. Anim. Sci., 23, 397-403.

Terroine E. F., VALla S., i933. Valeur comparée des différents aliments protéiques pour la croissance. Bull. Soc. Sci. d'Hyg. aliment., 21, ro5-174.

Vermorel M., Keller J., r967. Utilisation énergétique, par le Rat en croissance, des principales céréales composant des régimes isoazotés et équilibrés en acides aminés. Ann. Zootech., 16, 223-234. 